\title{
Progressive bilateral ocular ischemic syndrome despite previously performed carotid endarterectomy
}

\author{
Marta Świerczyńska $\odot^{1,2}$, Lech Sedlak $\oplus^{1,2}$, Mariola Dorecka $\oplus^{1,2}$ \\ 'Department of Ophthalmology, Faculty of Medical Sciences in Katowice, Medical University of Silesia, Katowice, Poland \\ ${ }^{2}$ Department of Ophthalmology, Kornel Gibiński University Clinical Center, Medical University of Silesia, Katowice, Poland
}

\begin{abstract}
The ocular ischemic syndrome (OIS) is a rare condition with vision-threatening potentials. It is characterized by chronic ischemia of the anterior and/or posterior segment of the eye primarily caused by severe carotid artery occlusive diseases. In this article, we present a case of a 67-year-old male patient presented with the gradual diminution of vision in the right eye for 6 months. The patient had undergone a right internal carotid artery (ICA) endarterectomy (CEA) twice before. On the day of admission, intraocular pressure (IOP) was $34 \mathrm{~mm} \mathrm{Hg}$ in the right eye (RE) and $20 \mathrm{~mm} \mathrm{Hg}$ in the left eye (LE). On anterior segment examination, neovascularization of iris in the right eye was noted. On fundoscopy dilated retinal veins, attenuated retinal arteries, blot hemorrhages, cotton wool spots and microaneurysms were present. The patient underwent the appropriate investigations including computed tomography angiography (CTA) of carotid arteries, fundus fluorescence angiography (FFA) and Doppler ultrasound (DUS) of ophthalmic and central retinal arteries, which were all consistent with a diagnosis of bilateral OIS. Intraocular pressure was pharmacologically reduced and stabilized. Subsequently, panretinal photocoagulation (PRP) of peripheral capillary nonperfusion areas was performed in both eyes.
\end{abstract}

KEY WORDS: bilateral ocular ischemic syndrome; IOS; carotid artery occlusion; neovascular glaucoma

Ophthalmol J 2020; Vol. 5, 114-119

\section{INTRODUCTION}

The ocular ischemic syndrome (OIS) is caused by arterial hypoperfusion of the eye due to severe stenosis or occlusion of the ipsilateral common or internal carotid artery (ICA) $[1,2]$. The reported incidence is 7.5 cases per million persons every year [3]. The average age of patients with OIS is 65 and it is rarely diagnosed among people before the age of 50 [2]. This condition is twice as common in men due to the higher incidence of cardiovascular and atherosclerotic diseases compared to women [3, 4]. Other risk factors include hypertension, hyperlipidemia, uncontrolled diabetes mellitus, smoking addiction and vasculitis [4]. Loss of vision is usually unilateral and progresses slowly. However, there are cases of sudden blindness [1]. In about $20 \%$ of cases, bilateral IOS occurs [3], which more often affects patients with the aortic arch syndrome, hyperhomocysteinemia and Takayasu arteritis $[2,5]$.

Visual loss of varying degree is the most common symptom [4]. Changes of the anterior segment include conjunctival and episcleral injection, rubeosis iridis, secondary neovascular glaucoma, iridocyclitis, iris atrophy, anterior and posterior synechiae, sluggish pupillary reaction to light, corneal edema with Descemet's folds, asymmetric cataract. More 
characteristic are changes related to the posterior segment, such as narrowed retinal arteries, dilated but not tortuous retinal veins, neovascularization of the optic disc and the retina, perifoveal telangiectasias, retinal hemorrhages, microaneurysms, cotton wool spots, areas of chorioretinal atrophy, macular edema or vitreous hemorrhage. These symptoms may co-occur with the orbital infarction syndrome, which includes ophthalmoplegia, orbital pain, hypotony and ptosis $[1,2,4,6]$.

The aim of this study was to present a case of a progressive bilateral ocular ischemic syndrome despite previously performed carotid endarterectomy.

\section{CASE REPORT}

A 67-year-old male presented to the emergency room complaining of recurrent unilateral eye pain and gradual decrease and blurring of the vision in his right eye for the past 6 months. His medical history included hypertension, coronary artery disease, hypercholesterolemia, type 2 diabetes mellitus and diabetic nephropathy.

He underwent artery bypass surgery nine years ago, percutaneous coronary intervention three years ago. He had twice undergone an ICA endarterectomy (in 2017 and 2018). There was no history of ocular or head trauma.

At the time of presentation, the best-corrected visual acuity (BCVA) was 5/50 in the RE and 5/8 in the LE. Anterior segment examination showed conjunctival injection and anterior synechiae in both eyes. The pupil in the RE was semi-dilated and non-reactive. Moreover, in the RE, there was advanced iris neovascularization and the presence of new vessels in the iridocorneal angle on gonioscopy.

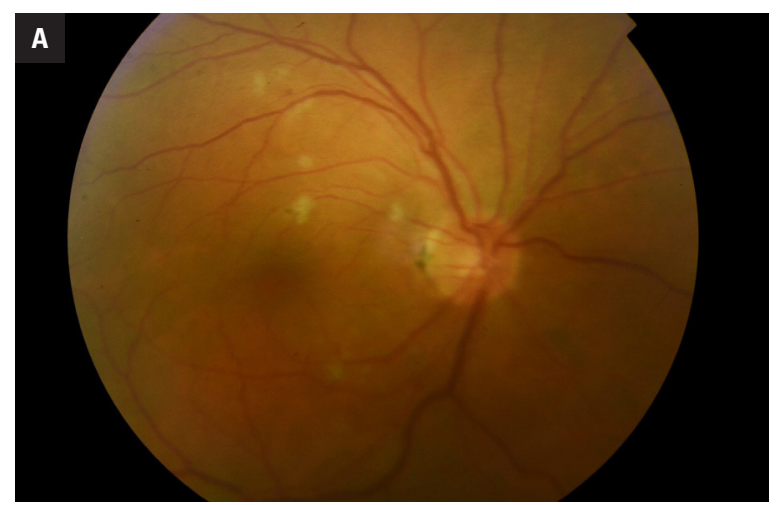

Fundus examination in both eyes showed attenuated retinal arteries, dilated retinal veins, cotton-wool spots, microaneurysms and blot hemorrhages at the retinal mid-periphery (Fig. 1). Intraocular pressure was $34 \mathrm{~mm} \mathrm{Hg}$ in the RE and $20 \mathrm{~mm} \mathrm{Hg}$ in the LE on applanation tonometry. The patient was admitted to the Department of Ophthalmology to reduce IOP and for further work-up.

Computed tomography angiography (CTA) of carotid arteries revealed critical narrowing (over $90 \%$ ) of the proximal segment of the right internal and external carotid arteries, confirming that the patient had developed carotid artery restenosis after his previous carotid endarterectomies (CEAs). Moreover, the CTA showed narrowing $60 \%$ of the proximal segment of the left ICA and occlusion of the right vertebral artery. There were no changes inside the orbital cavities that could lead to pressure on the blood vessels and ischemia of the eyeballs for reasons other than atherosclerosis.

Fundus fluorescence angiography (FFA) of the $\mathrm{RE}$ revealed prolonged arm-to-choroid and arm-to-retina circulation time, arteriovenous transit time delay, extensive non-perfusion areas within the peripheral retina, staining of the retinal vessels at the late phase and hyperfluorescence of the optic disc (Fig. 2). In the LE, non-perfusion zones in the temporal quadrants and macular edema were seen. Microaneurysms were also noted in both eyes (Fig. 3). This picture suggested diabetic retinopathy of both eyes. However, significant asymmetry of ischemic changes more strongly marked in the RE indicated OIS.

Doppler ultrasound (DUS) examination showed high retrograde flows in both ocular arteries (OA) (Fig. 4). Whereas in the central retinal arteries, the

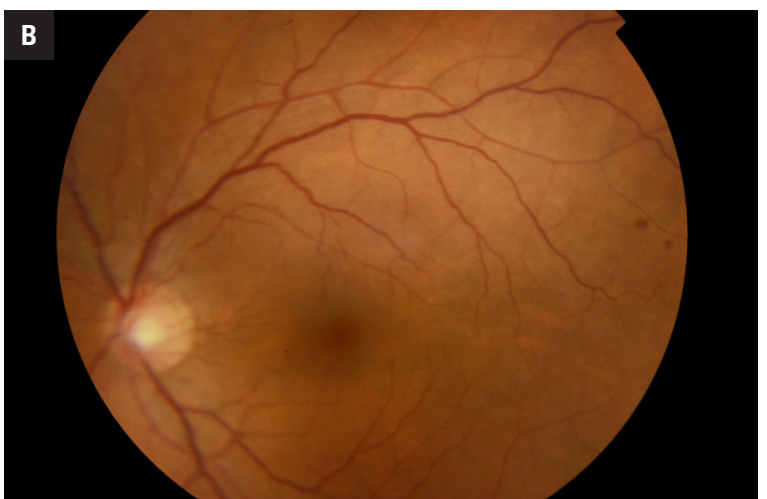

FIGURE 1AB. Fundus photographs of the right and left eye: dilated but not tortuous retinal veins, narrowed retinal arteries, cotton wool spots, microaneurysms and retinal hemorrhages at the mid-periphery 

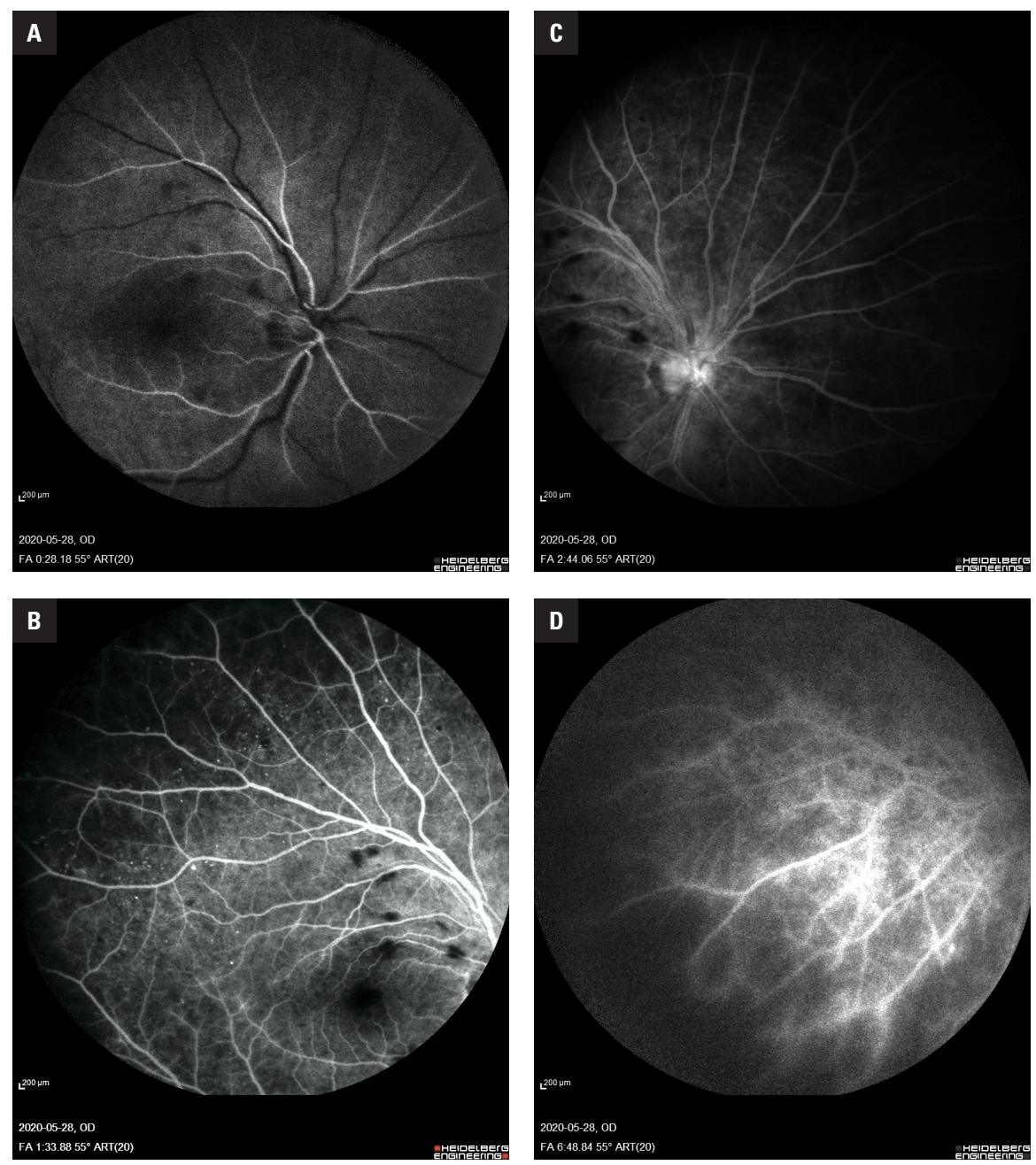

FIGURE 2A-D. AFF of the right fundus: delayed arterial phase (0:28), foci of blocked fluorescence from cotton-wool spots, hyperfluorescence of the microaneurysms, extensive non-perfusion zones at the periphery (1:33), hyperfluorescence of the optic disc, non-perfusion zones at the periphery $(2: 44)$, and staining of the retinal vessels at the late phase $(6: 48)$

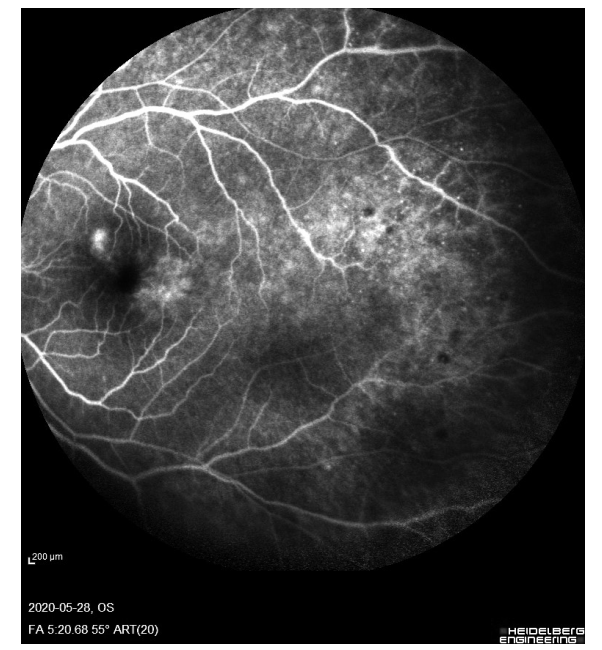

FIGURE 3. AFF of the left fundus: macular edema, microaneurysms, and peripheral non-perfusion zones (5:20) flows were disturbed, and the resistances increased (Tab. 1).

Due to the poor view of the fundus, optical coherence tomography (OCT) could not be performed. Complete blood count (CBC), erythrocyte sedimentation rate (ESR), C-reactive protein (CRP), coagulogram, and lipid profile were within normal limits. In contrast, fasting blood sugar (FBS) was $193 \mathrm{mg} / \mathrm{dL}$ (normal: 70-99 mg/dL), and glycated hemoglobin $\left(\mathrm{HBA}_{1 \mathrm{c}}\right.$ ) level was $6.8 \%$ (the norm in properly managed diabetes is up to $7 \%$ ).

Based on the above findings, the patient was diagnosed with bilateral OIS. Treatment with topical $\beta$-adrenergic blocker and $\alpha 2$-agonists along with carbonic anhydrase inhibitors was included, resulting in reduction and stabilization of IOP. Then, he was planned for panretinal photocoagulation (PRP) 

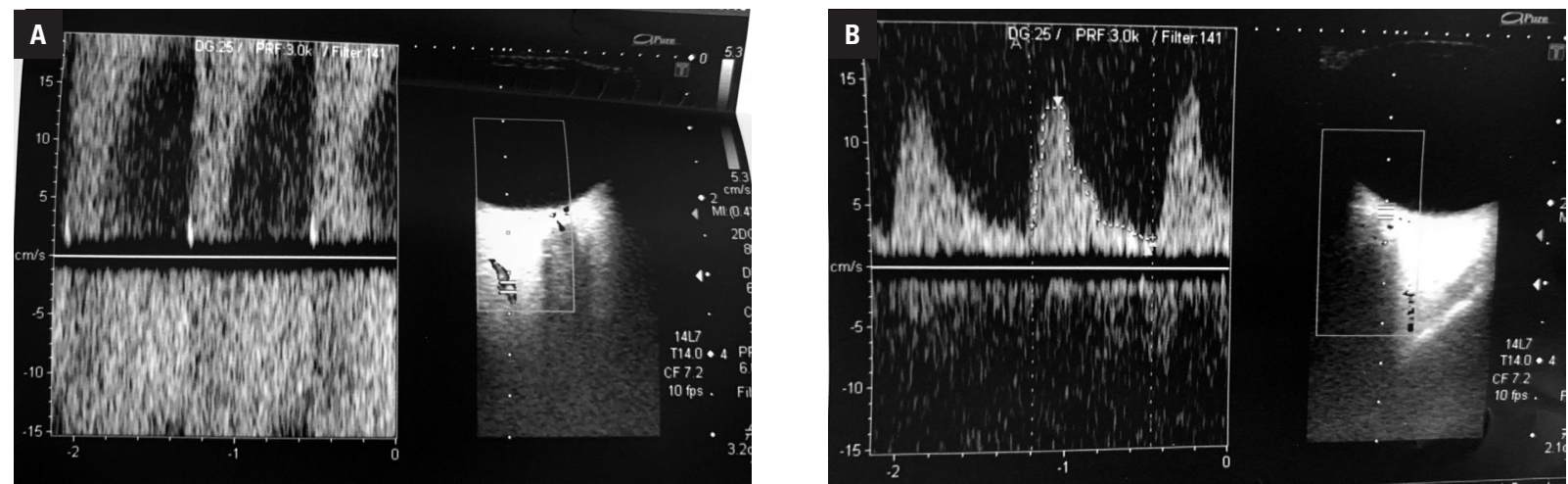

FIGURE 4. Color-coded duplex ultrasonography (Toshiba Xario, Model SSA-660A, Tokyo, Japan): A. Doppler signal of an inverted right ophthalmic artery flow due to right ICA stenosis (over $90 \%$ ) showing negative velocities; B. Doppler ultrasound (DUS) of right central retinal artery

\begin{tabular}{|c|c|c|}
\hline & RE & LE \\
\hline Vmax & $1.2 \mathrm{~cm} / \mathrm{s}$ & $12.9 \mathrm{~cm} / \mathrm{s}$ \\
\hline Vmin & $2.5 \mathrm{~cm} / \mathrm{s}$ & $2.8 \mathrm{~cm} / \mathrm{s}$ \\
\hline $\mathrm{PI}$ & 1.63 & 1.57 \\
\hline RI & 0.8 & 0.77 \\
\hline
\end{tabular}

$\mathrm{RE}$ — right eye; LE — left eye; Vmax — maximal velocity; Vmin — minimal velocity $\mathrm{Pl}$ — pulsatility index; $\mathrm{Rl}$ - resistance index

of peripheral capillary nonperfusion areas in both eyes. The procedure was performed in a typical way, without complications. The man was discharged from the hospital and informed about the need for further treatment of vascular changes as a matter of urgency by the vascular surgeon, cardiologist, neurologist due to the threat of vision in both eyes, as well as the diabetologist in order to stabilize blood glucose level. At the 3-month clinical follow-up, BCVA was still 5/50 in the RE and 5/8 in the LE.

\section{DISCUSSION}

In patients with OIS, a narrowing of the internal or common carotid arteries $90 \%$ or more is usually present on the same side as the symptoms. In half of the cases, complete occlusion is detected. Less often, the symptoms are caused by the occlusion of OA. There is a greater chance of developing OIS if the patient has poorly developed collateral circulation between the internal and external carotid arteries or between the two ICAs. In such circumstances, even a $50 \%$ stenosis can cause OIS $[1,6,7]$.

Atherosclerosis, progressing with age and/or due to dyslipidemia, is a major factor in sclerosis and stenosis of the carotid arteries resulting in ocular hypoperfusion $[1,2]$. Other causes include dissecting aneurysm of the carotid artery, aortic arch syndrome, giant cell arteritis, Takayasu arteritis, Behcet's disease, fibrovascular dysplasia, trauma or inflammation causing stenosis of the carotid arteries as well as complications after intravitreal anti vascular endothelial growth factor (anti-VEGF) injections or after radiotherapy for nasopharyngeal carcinoma $[1,3,8-10]$.

Often, asymptomatic onset (pain occurs in about $40 \%$ of cases) [1], as well as a wide and complex spectrum of ophthalmic symptoms causes that many patients with OIS remain undiagnosed or misdiagnosed, which may later contribute to irreversible loss of vision, as well as increased mortality $[2,3,6]$. Imaging studies of the carotid arteries play a key role in diagnosing OIS. Among them, the most common and non-invasive method is DUS of carotid arteries. CTA or, less often, magnetic resonance angiography are also used. Moreover, a useful test is DUS of retrobulbar vessels, in which decreased or reversed blood flow in the OA can be seen. Reversed blood flow in the OA arises when, as a result of the coexistence of severe ICA stenosis and incomplete blood circulation in the circle of Wills, the blood flow in the OA reverses to supply the ipsilateral brain. It is otherwise referred to as "steal-phenomenon" and leads to reduced retrobulbar blood flow, hypoperfusion and ischemia of ocular tissues. It is a highly specific indicator of ipsilateral stenosis or occlusion of ICA $[1,6]$.

Another test used in the diagnosis of OIS is FFA, where delayed arm-to-choroid and arm-to-retina circulation time is a common symptom. In almost $60 \%$ of cases, patchy or delayed choroidal 
filling time occurs, which is the most specific angiographic sign of OIS. However, the most sensitive angiographic sign of OIS (present in up to $95 \%$ of patients) is prolonged retinal arteriovenous time [1, 3]. Another common angiographic sign (observed in $85 \%$ of cases) is staining of the retinal vessels (primarily arteries) due to their increased permeability induced by endothelial cell damage because of chronic ischemia. In addition, hyperfluorescence of the optic disc is caused by leaks from disc capillaries. Moreover, cotton wool spots, edema of the nerve fiber layer of the optic disc and choroidal atrophy can also appear due to choroidal vascular compromise [1]. FFA can show the presence of microaneurysms around the macula or at the mid-periphery [11], as well as retinal capillary non-perfusion found mostly at the mid-periphery [3]. Indocyanine green angiography (ICG) is also helpful in assessing choroidal vascular abnormalities in eyes with OIS $[1,2]$. Furthermore, enhanced depth imaging optical coherence tomography (EDI-OCT) shows choroidal thinning and smaller luminal and stromal area in people with OIS or with significant stenosis of the carotid arteries [6, 12].

Differential diagnosis of IOS should primarily include moderately advanced central retinal vein occlusion (CRVO) and diabetic retinopathy (DR), then the hyperviscosity syndromes or autoimmune uveitis. Absence of tortuous retinal vein and sometimes retinal artery pulsations present in OIS help to distinguish it from CRVO. Cotton wool spots can be found in both OIS and DR. However, more intraretinal hemorrhages and the presence of hard exudates suggest diabetic etiology, while it should be borne in mind that DR and OIS may co-occur. Therefore, patients with asymmetric retinopathy should be examined for possible carotid artery stenosis. During FFA, the lack of retinal arterial stasis and choroidal filling defects in CRVO and DR allows them to be differentiated from OIS $[2,3]$.

The most dangerous effect of chronic retinal and choroidal ischemia is the increased production of VEGF, which causes neovascularization in the anterior (iris, iridocorneal angle) as well as posterior segment (retina, optic disc). Changes in the anterior segment may lead to the development of neovascular secondary glaucoma. At the early stages of glaucoma, topical $\beta$-adrenergic blockers or $\alpha 2$-agonists along with carbonic anhydrase inhibitors are mainly used to reduce IOP. Prostaglandin analogues, however, due to their potential pro-inflammatory effects, should not be used when neovascularization is accompanied by inflammation. Pilocarpine is also not recommended, as it may lead to posterior synechiae and increase occlusion of the iridocorneal angle. Moreover, it is also recommended to use mydriatics. [1, 2]. Trabeculectomy should be considered for patients not responding to drug treatment, with limited angle neovascularization and preserved VA. If this procedure is ineffective or significant angle neovascularization is present, it is recommended to implant aqueous shunt implants $[2,13]$. In the posterior segment, new vessels are more often formed at the optic disc than in the retina. They can cause bleeding, result in hemorrhages into the vitreous body and fibrovascular proliferation. To inhibit the formation of new vessels, PRP is performed. However, it should be noted that the effectiveness of this procedure is only $36 \%$ (because choroidal ischemia alone, without accompanying retinal ischemia, can induce neovascularization) and it does not show any promising effect in the visual outcome $[1,2]$. Moreover, there are studies on the use of anti-VEGF injections to treat neovascular glaucoma and macular edema associated with OIS [14].

Surgical treatments for carotid artery stenosis include CEA, which is $70-90 \%$ effective for symptomatic and $60 \%$ for asymptomatic patients [15]. An alternative treatment method is carotid artery stenting (CAS). However, with total occlusion, arterial by-pass surgery remains the only treatment option $[1,16]$. Restoration of normal blood flow, especially before the onset of iris neovascularization and secondary glaucoma, allows stabilization of existing VA. Performing the surgery at an early stage of the development of neovascularization may in turn contribute to the regression of changes that have already developed at the angle. However, it should be borne in mind that after revascularization surgery, as a result of increased oxygen distribution and re-production of aqueous humor by the ciliary body, IOP may increase $[2,3,16]$. The case described by us shows that despite proven high effectiveness, CEA is not always able to protect the patient from restenosis and OIS, which other authors have also encountered $[17,18]$.

In $29 \%$ of patients, symptomatic carotid stenosis co-occurs with changes in the retinal arteries, often without any symptoms, and $1.5 \%$ per year progress to symptomatic OIS. On the other hand, it is assumed that OIS may occur as the first symptom of ICA occlusion in $69 \%$ of patients [19]. According to Hayreh et al. [20], the incidence of myocardial ischemia before or after the onset of 
OIS was $22 \%$, whereas the incidence of transient ischemic attack (TIA)/stroke was $17 \%$. Furthermore, given that the 5 -year survival rate in patients with OIS is only $60 \%[4,21]$, it is extremely important for patients with OIS to have a neurological, cardiological and vascular surgical consultation as soon as possible to minimize the risk of morbidity and mortality.

\section{CONCLUSION}

OIS is a rare condition associated with systemic diseases and can result in irreversible vision loss as well as increased mortality rate. In the case where OIS is the first symptom of significant stenosis of the carotid arteries, the role of an ophthalmologist is extremely important and consists not only in making the right and early diagnosis and initiating appropriate treatment but also in cooperation with other specialists to prevent cerebrovascular and cardiovascular complications.

\section{Acknowledgements}

None.

\section{Statement of competing interests}

The authors declare that they have no conflict of interest.

\section{Ethics approval and consent to participate}

Ethics approval and consent to participate are not applicable in this case report.

\section{REFERENCES}

1. Mendrinos E, Machinis TG, Pournaras CJ. Ocular ischemic syndrome. Surv Ophthalmol. 2010; 55(1): 2-34, doi: 10.1016/j.survophthal.2009.02.024, indexed in Pubmed: 19833366.

2. Terelak-Borys B, Skonieczna K, Grabska-Liberek I. Ocular ischemic syndrome - a systematic review. Med Sci Monit. 2012; 18(8): RA138RA144, doi: $10.12659 / \mathrm{msm} .883260$, indexed in Pubmed: 22847215.

3. Sharma S, Brown GC. Ocular Ischemic Syndrome. In: Ryan SJ, Hinton DR, Schachat AP. ed. Retina. Elsevier, New York 2006: 1491-1502.

4. Luo J, Yan Z, Jia Yu, et al. Clinical Analysis of 42 Cases of Ocular Ischemic Syndrome. J Ophthalmol. 2018; 2018: 2606147, doi: 10.1155/2018/2606147, indexed in Pubmed: 29713523.

5. Hong IH, Ahn JK, Chang S, et al. Diagnostic efficacy of total homocysteine and C-reactive protein for ocular ischemic syndrome. Eye
(Lond). 2011; 25(12): 1650-1654, doi: 10.1038/eye.2011.232, indexed in Pubmed: 21921951.

6. Wang H, Wang Y, Li H. Multimodality Imaging Assessment of Ocular Ischemic Syndrome. J Ophthalmol. 2017; 2017: 4169135, doi: 10.1155/2017/4169135, indexed in Pubmed: 29375911.

7. Mizener J, Podhajsky P, Hayreh S. Ocular Ischemic Syndrome. Ophthalmology. 1997; 104(5): 859-864, doi: 10.1016/s01616420(97)30221-8.

8. Peter J, David S, Danda D, et al. Ocular manifestations of Takayasu arteritis: a cross-sectional study. Retina. 2011; 31(6): 1170-1178, doi: 10.1097/IAE.0b013e3181fe540b, indexed in Pubmed: 21317836.

9. Tang $Y$, Luo D, Peng W, et al. Ocular ischemic syndrome secondary to carotid artery occlusion as a late complication of radiotherapy of nasopharyngeal carcinoma. J Neuroophthalmol. 2010; 30(4): 315-320, doi: 10.1097/WN0.0b013e3181dee914, indexed in Pubmed: 20736842.

10. Huang $\mathrm{ZL}$, Lin $\mathrm{KH}$, Lee $\mathrm{YC}$, et al. Acute vision loss after intravitreal injection of bevacizumab (avastin) associated with ocular ischemic syndrome. Ophthalmologica. 2010; 224(2): 86-89, doi: 10.1159/000235726, indexed in Pubmed: 19707032.

11. Chen CS, Miller NR. Ocular ischemic syndrome: review of clinical presentations, etiology, investigation, and management. Compr Ophthalmol Update. 2007; 8(1): 17-28, indexed in Pubmed: 17394756.

12. Kang HM, Choi JH, Koh HJ, et al. Significant changes of the choroid in patients with ocular ischemic syndrome and symptomatic carotid artery stenosis. PLoS One. 2019; 14(10): e0224210, doi: 10.1371/ journal.pone.0224210, indexed in Pubmed: 31639146.

13. Wierzbowska J, Robaszkiewicz J, Figurska M, et al. Future possibilities in glaucoma therapy. Med Sci Monit. 2010; 16(11): RA252-RA259, indexed in Pubmed: 20980972.

14. Choi EY, Lee SC, Kim M. Optical Coherence Tomography Angiography Before and After Bevacizumab Injection in Ocular Ischemic Syndrome. Retina. 2018; 38(3): e23-e25, doi: 10.1097/IAE.0000000000001981, indexed in Pubmed: 29206756.

15. Dzierwa K, Pieniazek P, Musialek P, et al. Treatment strategies in severe symptomatic carotid and coronary artery disease. Med Sci Monit. 2011; 17(8): RA191-RA197, doi: 10.12659/msm.881896, indexed in Pubmed: 21804476.

16. Kawaguchi S, lida Jl, Uchiyama Y. Ocular Circulation and Chronic Ocular Ischemic Syndrome before and after Carotid Artery Revascularization Surgery. J Ophthalmol. 2012; 2012: 350475, doi: 10.1155/2012/350475, indexed in Pubmed: 23316337.

17. Tripathy K, Mazumdar S. Recurrent retinal and choroidal ischemia in a case of ocular ischemic syndrome. Ther Adv Ophthalmol. 2019; 11: 2515841419848926, doi: 10.1177/2515841419848926, indexed in Pubmed: 31321381.

18. Fintelmann RE, Rosenwasser RH, Jabbour $\mathrm{P}$, et al. An old problem, a new solution. Surv Ophthalmol. 2010; 55(1): 85-88, doi: 10.1016/j. survophthal.2009.03.005, indexed in Pubmed: 19726066.

19. Klijn CJM, Kappelle LJ, van Schooneveld MJ, et al. Venous stasis retinopathy in symptomatic carotid artery occlusion: prevalence, cause, and outcome. Stroke. 2002; 33(3): 695-701, doi: 10.1161/ hs0302.104619, indexed in Pubmed: 11872890.

20. Hayreh SS, Zimmerman MB. Ocular arterial occlusive disorders and carotid artery disease. Ophthalmol Retina. 2017; 1(1): 12-18, doi: 10.1016/j.oret.2016.08.003, indexed in Pubmed: 28547004.

21. Mills RP. Anterior segment ischemia secondary to carotid occlusive disease. J Clin Neuroophthalmol . 1989; 9(3): 200-204, indexed in Pubmed: 2529282. 